\title{
GROUP CUBIZATION
}

\author{
DAMIAN OSAJDA \\ WITH AN APPENDIX BY MIKAËL PICHOT
}

\begin{abstract}
We present a procedure of group cubization: it results in a group whose some features resemble the ones of a given group, and which acts without fixed points on a CAT(0) cubical complex. As a main application we establish lack of Kazhdan's property $(\mathrm{T})$ for Burnside groups.
\end{abstract}

\section{INTRODUCTION}

The initial motivation for the current article was the following well-known question:

Do all Burnside groups have Kazhdan's property (T)?

(See e.g. BdlHV01, Open Problem 17, page 9], the book BdlHV08, Open Example 7.3, page 282], or [Sha06, page 1304], and [Sha06a, Conjecture]. In the latter two, Y. Shalom presents it as a conjecture and provides a motivation.)

Recall that infinite Burnside groups, that is, finitely generated groups of bounded torsion were first constructed by Novikov-Adyan [NA68]. The free Burnside group $B(m, n)$ is defined by the presentation

$$
\left\langle s_{1}, s_{2}, \ldots, s_{m} \mid w\left(s_{1}, s_{2}, \ldots, s_{m}\right)^{n}\right\rangle,
$$

where $w$ runs over all words in $s_{1}, s_{2}, \ldots, s_{m}$. We answer in the negative the above question by proving the following.

Theorem 1. If the free Burnside group $B(m, n)$ is infinite then, for every integer $k>1$, the free Burnside group $B(m, k n)$ acts without fixed points on a CAT(0) cubical complex and hence does not have Kazhdan's property (T).

Instytut Matematyczny, Uniwersytet WrocŁawski, Pl. Grunwaldzki 2/4, 50-384 Wroceaw, Poland

Institute of Mathematics, Polish Academy of Sciences, Śniadeckich 8, 00656 Warszawa, POLAND

E-mail address: dosaj@math.uni.wroc.pl.

2010 Mathematics Subject Classification. 20F65, 20F50, 22D10.

Key words and phrases. Burnside group, Kazhdan's property (T), CAT(0) cubical complex. 
As the main tool we introduce a general procedure called group cubization 1 It is a simple trick interesting on its own, that we believe may be of broad use. It works as follows.

Let $G$ be a finitely generated group. Let $\widetilde{\Gamma}$ be the $\mathbb{Z}_{k}$-homology cover of a Cayley graph $\Gamma$ of $G$, where $\mathbb{Z}_{k}$ is the group $\mathbb{Z} / k \mathbb{Z}$ of integers modulo $k$. The covering graph $\widetilde{\Gamma}$ is equipped with a structure of a space with walls, defined by preimages of edges in $\Gamma$. We prove the following.

Theorem 2. For a finitely generated group $G$ and its Cayley graph $\Gamma$, the $\mathbb{Z}_{k}$-homology cover $\widetilde{\Gamma}$ of $\Gamma$ is a Cayley graph of a finitely generated group $\widetilde{G}$. If $G$ is infinite then $\widetilde{G}$ acts with unbounded orbits on a CAT(0) cubical complex.

The group $\widetilde{G}$ is called a cubization of $G$. Theorem 1 follows easily from Theorem 2 since a cubization $\widetilde{G}$ of a Burnside group $G$ is a Burnside group (of exponent multiplied by $k$ ).

In the rest of the paper, after some preliminaries (Section 2) we prove (in Section 31) Theorem 2, then Theorem 1, and finally we make some remarks on further applications of group cubizations (Section 44).

In an appendix by Mikaël Pichot an alternative proof of a result similar to Theorem 1 is presented.

Acknowledgments. I thank Światosław R. Gal, Tadeusz Januszkiewicz, Damian Sawicki, Daniel T. Wise, and the anonymous referees for remarks leading to improvements in the article. Parts of the paper were written while visiting McGill University. I would like to thank the Department of Mathematics and Statistics of McGill University for its hospitality during that stay. The research was partially supported by (Polish) Narodowe Centrum Nauki, grant no. UMO-2015/18/M/ST1/00050.

\section{Group cubization}

The results in this section use only few basic facts about covering spaces. A standard reference for those is e.g. Hat02].

Let $\Gamma=\operatorname{Cay}(\mathrm{G}, \mathrm{S})$ be a Cayley graph of a group $G$ generated by a finite symmetric set $S$ (that is, $S=S^{-1}$ ). We use the convention that every vertex belongs to two edges corresponding to a pair $\left\{s, s^{-1}\right\}$, for each $s \in S$. In particular, generators being involutions give rise to double edges, and the degree of vertices is $|S|$. Fix an integer $k>1$. Let $p: \widetilde{\Gamma} \rightarrow \Gamma$ be the $\mathbb{Z}_{k}$-homology covering of $\Gamma$, that is, the covering corresponding to the kernel $K$ of the natural map $\pi_{1}(\Gamma, 1) \rightarrow H_{1}\left(\Gamma ; \mathbb{Z}_{k}\right)=\bigoplus_{I} \mathbb{Z}_{k}$, where $I$ is the set indexing generators of $\pi_{1}(\Gamma, 1)$, and 1 is the vertex of $\Gamma$ being the identity of $G$. Observe that it is a characteristic covering, that is, $K$ is a characteristic

\footnotetext{
${ }^{1}$ We chose the term "cubization" as an analogue of "hyperbolization" Gro87. Both procedures modify the object while preserving some of its features. In contrast, "cubulation" (see e.g. Wis11,Wis12] ) equips a given object with an additional structure.
} 
subgroup of $\pi_{1}(\Gamma, 1)$. This subgroup can be identified with $\pi_{1}(\widetilde{\Gamma}, \widetilde{1})$, where $\widetilde{1}$ is a vertex with $p(\widetilde{1})=1$. Therefore, for any automorphism $g$ of $\Gamma$, we have $g_{*} \circ p_{*}\left(\pi_{1}(\widetilde{\Gamma}, \widetilde{1})\right)=p_{*}\left(\pi_{1}(\widetilde{\Gamma}, \widetilde{1})\right)$, and hence $g \circ p: \widetilde{\Gamma} \rightarrow \Gamma$ can be lifted to a map $\widetilde{g}: \widetilde{\Gamma} \rightarrow \widetilde{\Gamma}$ satisfying $p \circ \widetilde{g}=g \circ p$.

Every element $g \in G$ defines an automorphism of $\Gamma$ given by left multiplication by $g$ (and also denoted by $g$ ). Let $\widetilde{G}$ denote the set of all lifts of all such automorphisms.

Theorem 3. The set $\widetilde{G}$ forms a group generated by a set $\widetilde{S}$ with $|\widetilde{S}|=|S|$, and with $\widetilde{\Gamma}$ being its Cayley graph $\operatorname{Cay}(\widetilde{G}, \widetilde{S})$.

Proof. Observe that for any automorphism $g$ of $\Gamma$ its lift is entirely determined by the value on any vertex of $\widetilde{\Gamma}$. Therefore, $\widetilde{G}$ is a group and acts transitively on $\widetilde{\Gamma}$.

For $g \in G$, if its lift $\widetilde{g}$ fixes a vertex $\widetilde{v} \in \widetilde{\Gamma}$ then $g(p(\widetilde{v}))=p(\widetilde{v})$. Since $G$ acts freely on $\Gamma$, it follows that $g=1$, and hence $\widetilde{g}$ is a deck transformation. As $\widetilde{g}$ fixes a vertex, it is the identity on $\widetilde{\Gamma}$. Therefore, $\widetilde{G}$ acts freely on $\widetilde{\Gamma}$.

From the Sabidussi theorem Sab58] it follows now that $\widetilde{\Gamma}$ is the Cayley $\operatorname{graph} \operatorname{Cay}(\widetilde{G}, \widetilde{S})$, for some $\widetilde{S}$. Since the degree of vertices in $\Gamma$ is equal to the degree of vertices in $\widetilde{\Gamma}$, and they are both equal to cardinalities of the corresponding generating sets, we have $|S|=|\widetilde{S}|$.

Definition. The group $\widetilde{G}$ is called the cubization of a group $G$ with respect to its Cayley graph $\Gamma=\operatorname{Cay}(\mathrm{G}, \mathrm{S})$.

Choose a vertex $\widetilde{1} \in \widetilde{\Gamma}$ with $p(\widetilde{1})=1 \in \Gamma$. For every $s \in S$ we may choose its lift $\widetilde{s}$ such that $\widetilde{s}(\widetilde{1})$ is a vertex adjacent to $\widetilde{1}$. The set $\widetilde{S}=\{\widetilde{s} \mid s \in S\}$ of such lifts is a generating set of $\widetilde{G}$. We use it below.

Lemma 4. Let $g_{1}, \ldots, g_{n} \in G$ be such that $g_{1} g_{2} \cdots g_{n}={ }_{G} 1$. For $i=$ $1, \ldots, n$, let $\widetilde{g}_{i}$ be a lift of $g_{i}$. Then $\left(\widetilde{g}_{1} \cdots \widetilde{g}_{n}\right)^{k}=\mathrm{id}_{\widetilde{\Gamma}}$.

Proof. The set of deck transformations of $p: \widetilde{\Gamma} \rightarrow \Gamma$ is a group isomorphic to $\pi_{1}(\Gamma, 1) / K$, i.e., to $H_{1}\left(\Gamma, \mathbb{Z}_{k}\right)=\bigoplus_{I} \mathbb{Z}_{k}$. Since the latter has exponent $k$, for every deck transformation $\widetilde{g}$ (that is when $p \circ \widetilde{g}=p$ ) we have $\widetilde{g}^{k}=\mathrm{id}_{\widetilde{\Gamma}}$. In particular, if $g_{1} \cdots g_{n}={ }_{G} 1$ then $\widetilde{g}_{1} \cdots \widetilde{g}_{n}$ is a deck transformation, and hence $\left(\widetilde{g}_{1} \cdots \widetilde{g}_{n}\right)^{k}=\mathrm{id}_{\widetilde{\Gamma}}$.

\section{Proofs of Theorem 1 and Theorem 2}

3.1. Proof of Theorem 2. By Theorem 3 we have that $\widetilde{\Gamma}$ is the Cayley graph $\operatorname{Cay}(\widetilde{G}, \widetilde{S})$. First, we consider the case when no edge of $\Gamma$ separates $\Gamma$. D. Wise [Wis11, Section 9] and [Wis12, Section 10.3] observed that the vertex set of the $\mathbb{Z}_{2}$-homology cover of a graph has a natural structure of a space with walls 2 Similar structure exists for the $\mathbb{Z}_{k}$-homology cover (see

\footnotetext{
${ }^{2}$ In fact, in Wis11 the result is set in the more general setting of CAT(0) cube complexes. The preprint was circulating since 2009 , and the results were presented e.g. during
} 
e.g. Khu14, discussion on page 57]): the preimage of every open edge of $\Gamma$ disconnects the cover $\widetilde{\Gamma}$ into $k$ connected components. Such a partition of vertices of $\widetilde{\Gamma}$ gives rise to $2^{k-1}-1$ partitions into two nonempty sets - these are walls in the space with walls $\left(\widetilde{\Gamma}^{(0)}, \mathcal{W}\right)$. Obviously, the group $\widetilde{G}$ acts on $\left(\widetilde{\Gamma}^{(0)}, \mathcal{W}\right)$ : for every generator $\widetilde{s} \in \widetilde{S}$ a wall corresponding to $e \in E$ is mapped by $\widetilde{s}$ to a wall corresponding to $s(e)$. Clearly, the action has orbits that are unbounded with respect to the wall pseudo-metric.

In the case when there exists an edge separating $\Gamma$, the translates (by $G$ ) of this edge define the space with walls $\left(\Gamma^{(0)}, \mathcal{W}^{\prime}\right)$. The $G$-action on $\left(\Gamma^{(0)}, \mathcal{W}^{\prime}\right)$ has unbounded orbits, and induces a $\widetilde{G}$-action on $\left(\Gamma^{(0)}, \mathcal{W}^{\prime}\right)$ with the same property.

Finally, by [Nic04,CN05] it follows that $\widetilde{G}$ acts with unbounded orbits on a corresponding $\mathrm{CAT}(0)$ cubical complex.

3.2. Proof of Theorem 1. Let $G=B(m, n)$ be an infinite free Burnside group 3 Let $\Gamma$ be its Cayley graph with respect to the (symmetric) generating set $S$, with $|S|=2 m$. By Theorem 3 , the cubization $\widetilde{G}$ of $G$ with respect to $\Gamma$ is a group with the Cayley graph $\widetilde{\Gamma}=\operatorname{Cay}(\widetilde{G}, \widetilde{S})$ being the $\mathbb{Z}_{k}-$ homology cover of $\Gamma$, for $\widetilde{S}=\{\widetilde{s} \mid s \in S\}$. For every word $w=\widetilde{s}_{1} \cdots \widetilde{s}_{l}$ we have that $\left(s_{1} \cdots s_{l}\right)^{n}=_{G} 1$ and hence, by Lemma 4, $w^{k n}=\widetilde{G} 1$. It follows that the cubization $\widetilde{G}$ is a Burnside group of exponent $k n$. By Theorem 2 the group $\widetilde{G}$ acts with unbounded orbits on a $\operatorname{CAT}(0)$ cubical complex. As $\widetilde{G}$ is a quotient of the free Burnside group $B(m, k n)$, the latter admits a similar action.

\section{Further APPLiCATIONS}

Theorem 2 may be used to establish the existence of unbounded actions on CAT(0) cubical complexes for other classes of groups. In particular we have the following.

Corollary 1. If a group defined by a presentation $\left\langle S \mid r_{1}, r_{2}, \ldots\right\rangle$ is infinite then the group with the presentation $\left\langle S \mid r_{1}^{k}, r_{2}^{k}, \ldots\right\rangle$ acts with unbounded orbits on a $C A T(0)$ cubical complex.

This applies to the class of groups defined by presentations in which relators are proper powers. Many important classical examples of groups belong here: generalized triangle groups, generalized von Dyck groups, groups given by Coxeter's presentations of types $(l, m \mid n, k),(l, m, n ; q)$, and $G^{m, n, p}$ (see e.g. Tho95] and references therein), or various small cancellation groups (see e.g. Wis11,Wis12 and references therein).

a conference at UQAM in April 2010. Independently, the same result has been shown for graphs in AGS12.

${ }^{3}$ See e.g. Cou14 for a description of the state of the art of the theory of infinite Burnside groups. 


\section{REFERENCES}

[AGŠ12] Goulnara Arzhantseva, Erik Guentner, and Ján Špakula, Coarse nonamenability and coarse embeddings, Geom. Funct. Anal. 22 (2012), no. 1, 22-36, DOI 10.1007/s00039-012-0145-z. MR2899681

[BdlHV01] Geometrization of Kazhdan's Property (T), Oberwolfach Rep. (2001). Abstracts from the mini-workshop held July 7-14, 2001; Organized by B. Bekka, P. de la Harpe, A. Valette; Report No. 29/2001.

[BdlHV08] Bachir Bekka, Pierre de la Harpe, and Alain Valette, Kazhdan's property (T), New Mathematical Monographs, vol. 11, Cambridge University Press, Cambridge, 2008. MR2415834

[CN05] Indira Chatterji and Graham Niblo, From wall spaces to CAT(0) cube complexes, Internat. J. Algebra Comput. 15 (2005), no. 5-6, 875-885, DOI 10.1142/S0218196705002669. MR2197811

[Cou14] Rémi Coulon, On the geometry of Burnside quotients of torsion free hyperbolic groups, Internat. J. Algebra Comput. 24 (2014), no. 3, 251-345, DOI 10.1142/S0218196714500143. MR3211906

[Gro87] Misha Gromov, Hyperbolic groups, Essays in group theory, Math. Sci. Res. Inst. Publ., vol. 8, Springer, New York, 1987, pp. 75-263. MR919829

[Hat02] Allen Hatcher, Algebraic topology, Cambridge University Press, Cambridge, 2002. MR1867354

[Khu14] Ana Khukhro, Embeddable box spaces of free groups, Math. Ann. 360 (2014), no. 1-2, 53-66, DOI 10.1007/s00208-014-1029-3. MR3263158

[Nic04] Bogdan Nica, Cubulating spaces with walls, Algebr. Geom. Topol. 4 (2004), 297-309 (electronic), DOI 10.2140/agt.2004.4.297. MR2059193

[NA68] Petr S. Novikov and Sergei I. Adyan, Infinite periodic groups. I, II, III, Izv. Akad. Nauk SSSR Ser. Mat. 32 (1968), 212-244, 251-524, 709-731 (Russian). MR0240178, MR0240179, MR0240180

[Sab58] Gert Sabidussi, On a class of fixed-point-free graphs, Proc. Amer. Math. Soc. 9 (1958), 800-804, DOI 10.2307/2033090. MR0097068

[Sha06] Yehuda Shalom, The algebraization of Kazhdan's property (T), International Congress of Mathematicians. Vol. II, Eur. Math. Soc., Zürich, 2006, pp. 12831310. MR2275645

[Sha06a] - Rigidity Theory of Discrete Groups, Lie Groups: Dynamics, Rigidity, Arithmetic; A conference in honor of the 60th birthday of Gregory Margulis (2006), available at http://people.brandeis.edu/ kleinboc/Margconf/shalom.pdf

[Tho95] Richard M. Thomas, Group presentations where the relators are proper powers, Groups '93 Galway/St. Andrews, Vol. 2, London Math. Soc. Lecture Note Ser., vol. 212, Cambridge Univ. Press, Cambridge, 1995, pp. 549-560, DOI 10.1017/CBO9780511629297.021. MR1337297

[Wis11] Daniel T. Wise, The structure of groups with quasiconvex hierarchy (2011), preprint, available at https://docs.google.com/open?id=0B45cNx80t5-2T0twUDFxVXRnQnc

[Wis12] From riches to raags: 3-manifolds, right-angled Artin groups, and cubical geometry, CBMS Regional Conference Series in Mathematics, vol. 117, Published for the Conference Board of the Mathematical Sciences, Washington, DC; by the American Mathematical Society, Providence, RI, 2012. MR2986461 


\section{Appendix: \\ A comment on Osajda's "Group cubization" paper}

Mikaël Pichot*

Answering a question of Shalom, Damian Osajda proved in the main body of this paper that the free Burnside group $B(m, k n)$, for $k \geq 2$, does not have the property ( $\mathrm{T})$ of Kazhdan if $B(m, n)$ is infinite.

Osajda's proof is a nice geometric argument using mod $k$ homology covers of Cayley graphs.

There is also a more algebraic argument for a similar result, which can implicitly be found in the literature. The goal of this appendix is to explain this alternative argument.

Consider the wreath product $W:=\mathbb{Z} / k \mathbb{Z}\} B(m, n)$ of $B(m, n)$ with a finite cyclic group. Notice that the order of every element in $W$ divides $k n$, and that $W$ is generated by $m+1$ elements. Therefore, by the universal property, $W$ is a quotient of the free Burnside group $B(m+1, k n)$ of exponent $k n$. It follows from [1, Theorem 3, p. 1897] or [3, Theorem 1.2, p. 168] that the wreath product $H<G$ does not have Kazhdan's property (T) if $G$ is infinite and $H$ is not trivial. In particular, the group $B(m+1, k n)$ does not have Kazhdan's property (T) if $B(m, n)$ is infinite. (Both the wreath product approach and Osajda's mod $k$ homology cover approach provide wall spaces in the over group, and therefore prove more than just the lack of property (T).)

The fact that nontrivial wreath product decompositions appear as quotients of large free Burnside groups was used in [2, Proof of Theorem 2] in relation with Dixmier's unitarizability problem.

The author is grateful to Nicolas Monod, Narutaka Ozawa, and Alain Valette for their comments.

\section{REFERENCES}

[1] P.-A. Cherix, F. Martin, and A. Valette. "Spaces with measured walls, the Haagerup property and property (T)". Ergodic theory and dynamical systems 24.06 (2004), pp. 1895-1908.

[2] N. Monod and N. Ozawa. "The Dixmier problem, lamplighters and Burnside group". Journal of Functional Analysis 258.1 (2010), pp. 255-259.

[3] M. Neuhauser. "Relative property (T) and related properties of wreath products". Mathematische Zeitschrift 251.1 (2005), pp. 167177 .

(*) Dept. of Mathematics \& Statistics, McGill University, Montréal, Québec, Canada H3A 2K6. pichot@math.mcgill.ca 